\title{
Spontaneous carotid-cavernous fistula with ophthalmoplegia and facial palsy
}

\author{
ANIL KAPUR \\ M.D. \\ N. K. PARIKH \\ M.B.B.S.
}

\author{
N. G. SANGHAVI \\ M.D.
}

S. K. AMIN

M.D.

Department of Medicine, Medical College and S.S.G. Hospital, Baroda, India

\section{Summary}

A hypertensive patient presenting with facial palsy and ophthalmoplegia following a spontaneous carotidcavernous fistula is presented. The mechanism of facial palsy in such cases is discussed.

\section{Introduction}

Spontaneous carotid-cavernous fistulae are uncommon. They are seen mostly in association with hypertension, atherosclerosis or intracranial aneurysm (Bickerstaff, 1970). Facial palsy was noted by Walsh (1957) in a case of traumatic carotid-cavernous fistula which recovered in one year. Involvement of the third, fourth, fifth and sixth cranial nerves has been frequently noted (Walsh, 1957; Sattler, 1957; Abrahamson and Bell, 1955). The present case report describes development of facial palsy and ophthalmoplegia following a spontaneous carotid-cavernous fistula.

\section{Case report}

A 44-year-old male presented complaining of something 'popping off' in the head on micturition (14 January 1980), following which he developed a roaring sound on the left side of the head. Next day he had an excruciating left-sided headache. Two days later he noticed excessive watering and prominence of the left eye ball. The next day he complained of double vision whilst his other symptoms worsened. $\mathrm{He}$ was admitted 5 days after the onset with a diagnosis of retrobulbar cellulitis, and the next day developed a left-sided facial palsy.

The patient was known to have hypertension and had been taking treatment for it. Physical examination revealed proptosis of the left eye with chemosis and conjuctival injection. Veins on the left side of the face were prominent especially over the forehead. The blood pressure was $150 / 110 \mathrm{mmHg}$. The left pupil was dilated and did not react to light but the indirect light reflex was present. There was complete ophthalmoplegia on the left side. Fundoscopy revealed grade I hypertensive retinopathy in both eyes; veins on the left were prominent. There was hyperaesthesia on the upper half of the face on the left. A left-sided infranuclear seventh nerve palsy with loss of taste sensation on the anterior two thirds of the tongue was noted. Tests for eighth nerve function were inconclusive. The caloric test was not done and no other neurological deficit was noted. A bruit was audible over the left eye ball and temporal region, which disappeared on compression of the left carotid artery. An ear, nose and throat examination carried out later to rule out a naso-pharyngeal mass was normal.

$\mathrm{X}$-rays of the skull and orbit were normal. A leftsided carotid angiogram done later revealed direct filling of the left cavernous sinus from the carotid artery. The superior orbital vein on the left side was outlined and there was early filling of the left jugular vein (Figs 1 and 2). This confirmed the diagnosis of a carotid-cavernous fistula.

The facial palsy recovered completely in 3 weeks, there was slight improvement in the ophthalmoplegia, and the congestion in the left eye regressed to some extent, while the proptosis and bruit persisted.

Due to the lack of neurosurgical facilities at this centre, the patient was referred to another centre where a carotid ligation was performed. The patient improved following the operation and at present has no neurological deficit. The noise in the ear has disappeared.

\section{Discussion}

Carotid-cavernous fistulae have varied modes of presentation, the most common being headache, roaring noise in the ear, pulsating exophthalmos and ophthalmoplegia. Bickerstaff (1970) has described other modes of presentation wherein a single fistula 

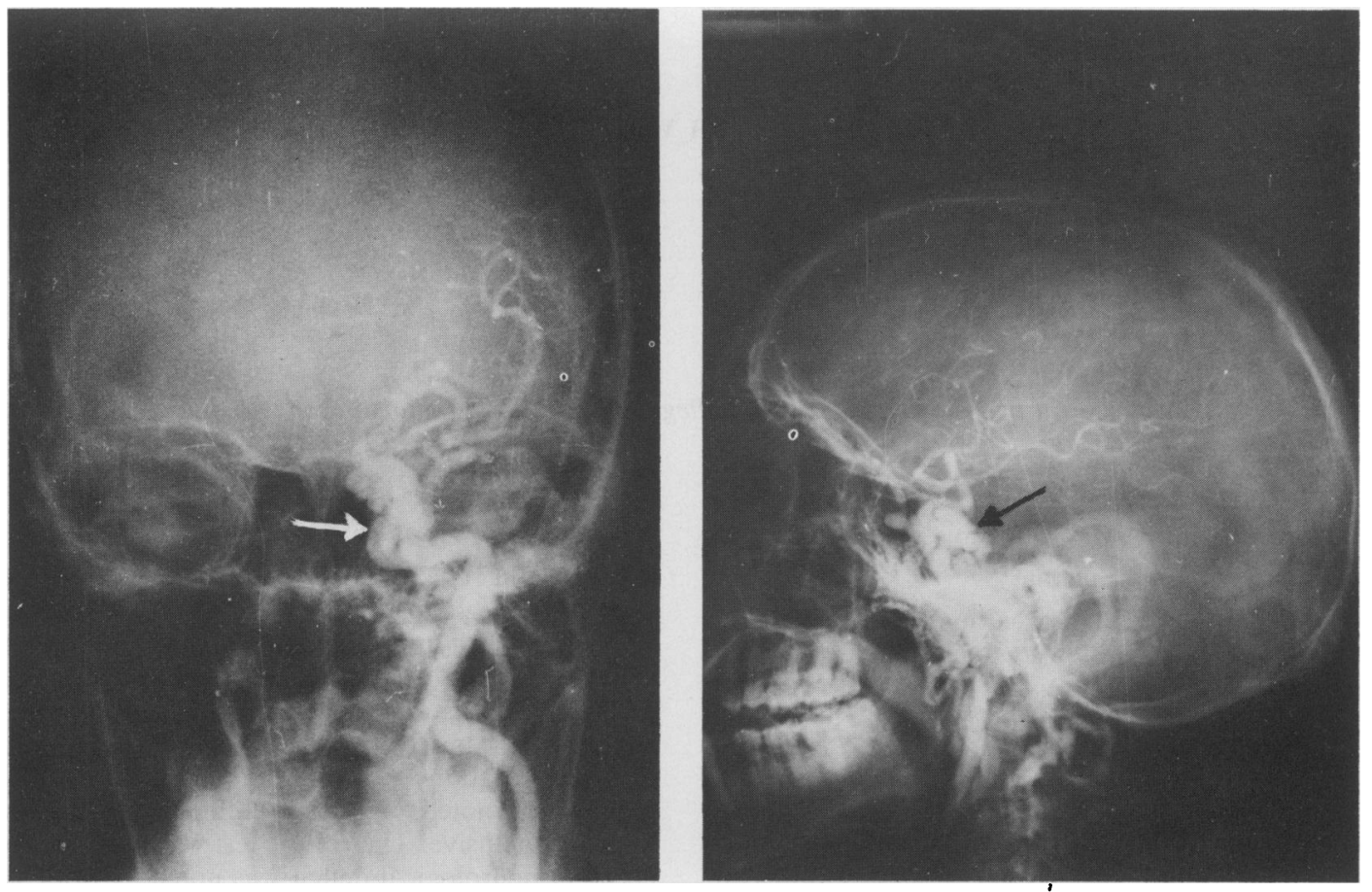

FIGS. 1 and 2. AP and lateral view of left-sided carotid angiogram showing direct filling of the left cavernous sinus from the carotid artery. The superior orbital vein on the left side is outlined and there is early filling of the left jugular vein.

may involve both sides or the fistula may cause involvement of the contralateral eye or both eyes alternatively.

Third, fourth and sixth cranial nerve palsies have been frequently noted and the mechanism of involvement described (Walsh, 1957; Sattler, 1957; Abrahamson and Bell, 1955). The ophthalmic division of the fifth nerve may also be affected and lead to corneal ulceration (Dandy and Follis, 1941). Papilloedema and optic atrophy secondary to either long standing papilloedema or glaucoma, have also been described (Bickerstaff, 1970; Sugar and Meyer, 1970). Optic atrophy may result from direct pressure of the carotid artery on the optic nerve (Dandy and Follis, 1941).

However, the authors have found only a solitary report of facial palsy associated with a traumatic arteriovenous aneurysm in a cavernous sinus (Walsh, 1977) which followed fracture of the skull; the facial palsy was noted immediately, recovery taking one year. No explanation for the facial nerve involvement was given. The present case had a left infranuclear facial palsy which occurred on the 6th day following a spontaneous carotid-cavernous fistula and recovered completely within 3 weeks. The most likely mechanism of facial involvement in the present case is a pressure palsy due to engorgement of the vein

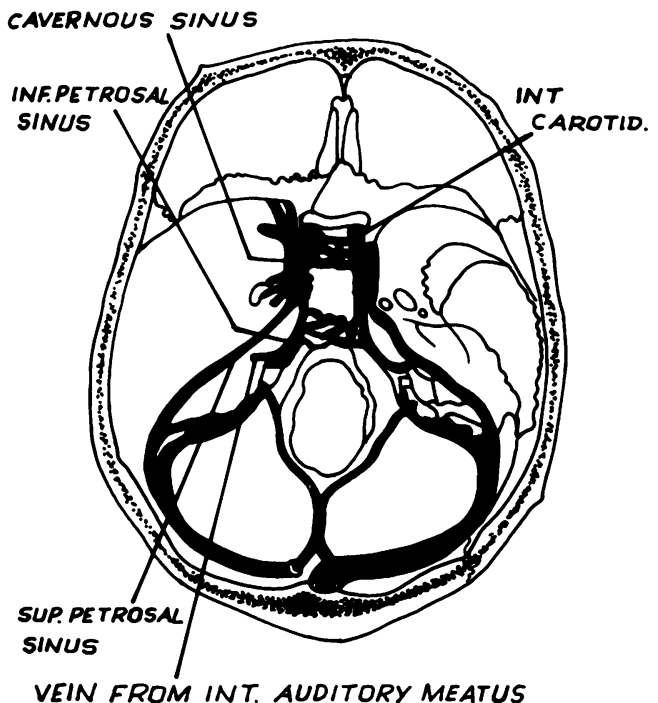

FIG. 3. Diagram showing the relation of vein from internal auditory meatus to inferior petrosal sinus. 
from the internal auditory meatus (Fig. 3). This vein drains blood into the inferior petrosal sinus. The latter is the main channel draining blood posteriorly into the jugular vein from the cavernous sinus and is likely to be greatly engorged in cases of carotid cavernous fistula. It is therefore surprising that the facial nerve is so uncommonly affected. Such a pressure palsy is likely to affect the eighth nerve also. It is possible that the present case may have had eighth nerve involvement but it could not be detected clinically as the loud bruit interfered with such testing. Moreover, symptoms of vestibular involvement like giddiness and nausea could be caused by the accompanying diplopia. That the facial palsy was due to congestion and oedema is further substantiated by its complete regression within 3 weeks.

An interesting fact demonstrated by the present case is that congestion in venous sinuses draining the cavernous sinus can also cause cranial nerve involve- ment. Also, it may be difficult to diagnose carotidcavernous fistula without a classical history when such extensive cranial nerve involvement is present, for it can be mistaken as a retro-orbital or a nasopharyngeal tumour.

\section{References}

Abrahamson, I.A. JR \& Bell, B.L. JR (1955) Carotid-cavernous fistula syndrome. American Journal of Ophthalmology, 39, 521.

BICKERSTAFF, E.R. (1970) Mechanism of presentation of carotid cavernous fistula. British Journal of Ophthalmology, 54, 186.

DANDY, W.E. \& FolLIS, R.H. (1941) On the pathology of carotidcavernous aneurysms. American Journai of Ophthalmology, 24, 365.

SATTLER, C.H. (1957) As quoted by Walsh (1957).

SUGAR, M.S. \& MEYER, S.S. (1970) Pulsating exophthalmos. Archives of Ophthalmology, 23, 1288.

WALSH, F.P. (1957) Vascular lesions and circulatory disorder of the nervous system. Clinical Neuro-opthalmology, 2nd Ed, pp. 850-861. William \& Wilkins, Baltimore. 